\title{
A comparative study of 43 patients with mirror- like intracranial aneurysms: risk factors, treatment, and prognosis
}

\author{
Rong Wangl,* \\ Dong Zhangl,* \\ Jizong Zhao' \\ Shuo Wang' \\ Yuanli Zhao' \\ Hongchuan $\mathrm{Niu}^{2}$ \\ 'Neurosurgical Department, Beijing \\ Tiantan Hospital affiliated to Capital \\ Medical University, ${ }^{2}$ Capital Medical \\ University, Beijing, People's Republic \\ of China \\ *These authors contributed equally \\ to this work
}

This article was published in the following Dove Press journal:

Neuropsychiatric Disease and Treatment

19 November 2014

Number of times this article has been viewed

Background and objective: Mirror-like intracranial aneurysms (MirAn) occur symmetrically at the corresponding intracranial arteries and are a subgroup of multiple intracranial aneurysms. The aim of this study was to analyze the risk factors, treatment, and prognosis of MirAn.

Methods: We retrospectively analyzed 43 cases of MirAn diagnosed between January 2000 and December 2009. The control groups comprised patients with non-mirror-like multiple aneurysms (nMirAn) and single aneurysms (SingAn). Sex, age, localization of MirAn, hypertension, diabetes, smoking, and rupture were identified as potential risk factors for MirAn.

Results: The male to female ratio of the MirAn patients was 1.0:5.1, which was significantly different from that of the nMirAn patients $(1.0: 1.9, P=0.037)$ and SingAn patients $(1.0: 1.3, P<0.001)$. There was no difference in age $(P=0.8741)$, smoking $(P=0.301)$, diabetes $(P=0.267)$, or hypertension $(P=0.874)$ between the MirAn and nMirAn patients. The aneurysms in 28 MirAn patients $(65.1 \%)$ involved the internal carotid-posterior communicating arteries; in these patients, the rupture risk was significantly higher for larger aneurysms compared with smaller aneurysms $(P<0.05)$.

Conclusion: More women suffered from MirAn than nMirAn or SingAn. The most common MirAn sites were the internal carotid-posterior communicating arteries. Our results suggest that MirAn was not associated with age, smoking, hypertension, or diabetes.

Keywords: women's health, mirror-like intracranial aneurysm, retrospective analysis, clinical feature

\section{Introduction}

Mirror-like intracranial aneurysms (MirAn) are a subgroup of multiple intracranial aneurysms that occur bilaterally on corresponding arteries. MirAn are rare and represent less than $5 \%$ of all intracranial aneurysms, but they account for as much as $40 \%$ of all multiple intracranial aneurysms. MirAn are usually found in the middle cerebral, pericallosal, and internal carotid arteries. ${ }^{1-3}$ Unruptured multiple intracranial aneurysms account for $20 \%-30 \%$ of all aneurysms that cause subarachnoid hemorrhage (SAH). ${ }^{3}$ The incidence of multiple intracranial aneurysms, particularly MirAn, is associated with a family history of aneurysms and with younger age; on the other hand, "classical" aneurysms occur later in life and are probably attributable to congenital weakness of the vessel wall in cases of MirAn. ${ }^{3}$

Only a few studies have examined the genetic predisposition to MirAn and other risk factors such as age, sex, hypertension, alcohol consumption, and smoking. Most of these studies are case reports, and they were therefore unable to confirm or refute any hypotheses. ${ }^{1,2,4-8}$ Therefore, studies in larger cohorts of MirAn patients are needed to confirm the association between MirAn and its supposed risk factors, since a better
Neurosurgical Department, Beijing

Tiantan Hospital affiliated to Capital Medical University, Beijing I00050,

People's Republic of China

Tel +861065 II 3440

Fax +861065113440

Email zhaojzl@I63.com 
understanding of the risk factors associated with MirAn could help with rupture prevention, diagnosis, treatment, and prognosis.

To explore these risk factors, we conducted a retrospective analysis of 43 patients who were diagnosed with MirAn. Age, sex, family history of aneurysms, risk factors for aneurysms (hypertension, smoking, alcohol consumption, and diabetes), and location of the aneurysms were compared between patients with MirAn, non-mirror-like multiple aneurysms (nMirAn), and single aneurysms (SingAn).

\section{Patients and methods}

\section{Patients}

We retrospectively assessed the medical records of 1,391 patients admitted with intracranial aneurysms between January 2000 and December 2009 at the Department of Neurosurgery at the Beijing Tiantan Hospital affiliated to Capital Medical University (Beijing, People's Republic of China). This study was approved by the ethical committee of the Capital Medical University of Beijing, and individual consent from participants was waived by the committee.

The following were the criteria for inclusion in the MirAn group: presence of twin bilateral intracranial aneurysms located at the corresponding contralateral arteries of the same name or the proximal part of a directly connecting artery, absence of symmetry or a specific direction of growth of the aneurysm, and absence of extracranial aneurysms.

All patients with multiple aneurysms who were not included in the MirAn group were included in the nMirAn group. All patients with a SingAn were included in the SingAn group.

Patients who had indications of SAH underwent brain $\mathrm{CT}$, and the SAH was graded according to the Hunt and Hess scale. ${ }^{9}$ After confirmation of SAH by CT, digital subtraction angiography (DSA) was performed. Patients with obvious arteriovenous malformations or Moyamoya disease were excluded.

\section{Treatment approach}

In the case of patients with unruptured aneurysms, the rupture risk was first explained to the patient, and then both the surgical and endovascular options were discussed with the patients and their families. After SAH was diagnosed by DSA, craniotomy was the first recommended treatment.

Patients who had a Hunt and Hess grade $\geq$ IV or comorbidities such as myocardial infarction, severe pulmonary infection, renal insufficiency, and coagulation abnormalities, and patients who were reluctant to undergo craniotomy underwent interventional embolization instead of aneurysm clipping. Patients who were smokers or suffered from hypertension were strongly encouraged to undergo further treatment.

Based on the CT scans, DSA, and clinical symptoms, the potential side of the ruptured aneurysm responsible for SAH was predicted and later confirmed during the surgery. The Glasgow outcome scale (GOS) was used to assess all the patients 6 weeks after they were admitted to the hospital. Patients were followed up for 12 months using outpatient clinical examination to determine their prognosis.

\section{Data collection and follow-up}

Patients and their family were questioned from medical history at admission. Age, sex, clinical features, and risk factors (hypertension, diabetes, and smoking) were compared between the three groups. Smoking was defined as more than five cigarettes per day or regular smoking for a year or more.

\section{Statistical analysis}

All statistical analyses were performed using SPSS 13.0 (SPSS Inc, Chicago, IL, USA). Categorical data are presented as proportions, and were analyzed using the chi-square or Fisher's exact tests, as appropriate. A $P$-value $<0.05$ was considered significant.

\section{Results}

\section{Patient characteristics}

Among 1,391 patients with intracranial aneurysms, 1,278 had SingAn and 113 had multiple aneurysms. In the SingAn group, the mean age was $46.7 \pm 11.5$ years; this group comprised 561 men and 717 women (male to female ratio, $1: 1.3$ ) (Table 1).

Among the patients with multiple intracranial aneurysms, 43 patients had mirror aneurysms and 70 patients had nonmirror aneurysms (MirAn to nMirAn ratio, 1.00:1.16). MirAn represented $3.1 \%$ of all intracranial aneurysms and $38.1 \%$ of all multiple intracranial aneurysms. In the MirAn group, the mean age was $51.4 \pm 9.2$ years; this group comprised seven men and 36 women (male to female ratio, 1.0:5.1). In the nMirAn group, the mean age was 51.3 \pm 9.9 years, and this group comprised 24 men and 46 women (male to female ratio, 1.0:1.9). Only one patient in the SingAn group suffered from Marfan syndrome (Table 1). Most patients presented with $\mathrm{SAH}$ ( $>80.0 \%$ in all groups). No patient was diagnosed incidentally in the MirAn group (Table 1), 
Table I Demographic characteristics according to the type of intracranial aneurysm

\begin{tabular}{|c|c|c|c|c|c|}
\hline \multicolumn{2}{|c|}{ Characteristic } & \multirow{2}{*}{$\begin{array}{l}\text { MirAn, n (\%) } \\
7(16.3)\end{array}$} & \multirow{2}{*}{$\begin{array}{l}\text { nMirAn, n (\%) } \\
24(34.3)\end{array}$} & \multirow{2}{*}{$\begin{array}{l}\text { SingAn, n (\%) } \\
56 \mathrm{I}(43.9)\end{array}$} & \multirow{2}{*}{$\frac{P \text {-value }}{0.0005^{*}}$} \\
\hline Sex & M & & & & \\
\hline & $\mathrm{F}$ & $36(83.7)$ & $46(65.7)$ & $717(56.1)$ & \\
\hline & $M: F$ & $\mathrm{I}: 5 . \mathrm{I}$ & I:1.9 & $1: 1.3$ & \\
\hline \multicolumn{2}{|c|}{ Age (years), mean $\pm S D$} & $51.4 \pm 9.2$ & $51.3 \pm 9.9$ & $46.7 \pm 11.5$ & $0.0002 *$ \\
\hline \multirow[t]{5}{*}{ Age groups } & $<30$ & 0 & 0 & $98(7.7)$ & $0.0022^{*}$ \\
\hline & $30-39$ & $7(16.3)$ & $9(12.9)$ & $208(16.3)$ & \\
\hline & $40-49$ & $10(23.3)$ & $17(24.3)$ & $442(34.6)$ & \\
\hline & $50-59$ & $16(37.2)$ & $32(45.7)$ & $363(28.4)$ & \\
\hline & $\geq 60$ & $10(23.2)$ & $12(17.1)$ & $167(13.1)$ & \\
\hline \multicolumn{2}{|c|}{ Marfan syndrome } & 0 & 0 & $\mathrm{I}(0.07)$ & \\
\hline \multirow[t]{3}{*}{ Presentatior } & $\mathrm{SAH}$ & $40(93.0)$ & $58(80.0)$ & I,203 (89.2) & \\
\hline & Neurologic symptoms" & $3(7.0)$ & $8(11.4)$ & $68(5.3)$ & \\
\hline & Incidental finding & 0 & $6(8.6)$ & $7(5.5)$ & \\
\hline
\end{tabular}

Notes: *Significant difference, $P<0.05$. Including oculomotor nerve palsy, diminution of vision, and visual field defect.

Abbreviations: MirAn, mirror-like intracranial aneurysms; nMirAn, non-mirror-like multiple aneurysms; SingAn, single aneurysms; M, male; F, female; SAH, subarachnoid hemorrhage; SD, standard deviation.

and incidental aneurysm findings were rare in the nMirAn and SingAn groups (Table 1).

Significantly more women had MirAn $(P=0.0005)$, while multiple intracranial aneurysms (both MirAn and nMirAn) occurred at an older age $(P=0.002)$ (Table 1$)$.

\section{Risk factor analysis}

There was no significant difference between these two groups with regard to hypertension, diabetes, and smoking (MirAn vs nMirAn $44.2 \%$ vs $45.7 \%, P=0.87 ; 7.0 \%$ vs $4.3 \%$, $P=0.27 ; 18.6 \%$ vs $27.1 \%, P=0.30$, respectively). Moreover, only one patient in the SingAn group had a family history of aneurysms. Stratification analysis of the MirAn and nMirAn patients by age group (Figure 1A and B, respectively) revealed that the proportion of patients with hypertension increased with age, but the difference between the age groups was not significant.

Among the 43 MirAn patients, three (7.0\%) had ruptured aneurysms and $40(93.0 \%)$ had induced SAH. Of the three patients who did not have ruptured aneurysms, two had blepharoptosis on one side and one had impaired vision. All three were outpatients and were diagnosed with aneurysms using magnetic resonance angiography and CT angiography. Their diagnoses were confirmed by DSA after admission to the hospital. Table 2 presents the clinical characteristics of these 43 MirAn patients. The left to right cerebral aneurysm ratio associated with the rupture was 1.00:1.11, which suggests that there was no dominant side.

To examine the effect of the size of the aneurysm on rupture, we compared the larger aneurysms with the smaller ones. Thirty aneurysms that were responsible for hemorrhage were larger ones, while eight patients had bleeding from the smaller aneurysms. The ratio of large to small hemorrhagic aneurysms was 3.75:1.00 $(P<0.01)$.

Among the 43 cases of MirAn, 28 (65.1\%) had aneurysms of the internal carotid-posterior communicating arteries, and $12(27.9 \%)$ had aneurysms of the middle cerebral arteries. One patient $(2.3 \%)$ had aneurysms of the internal carotid
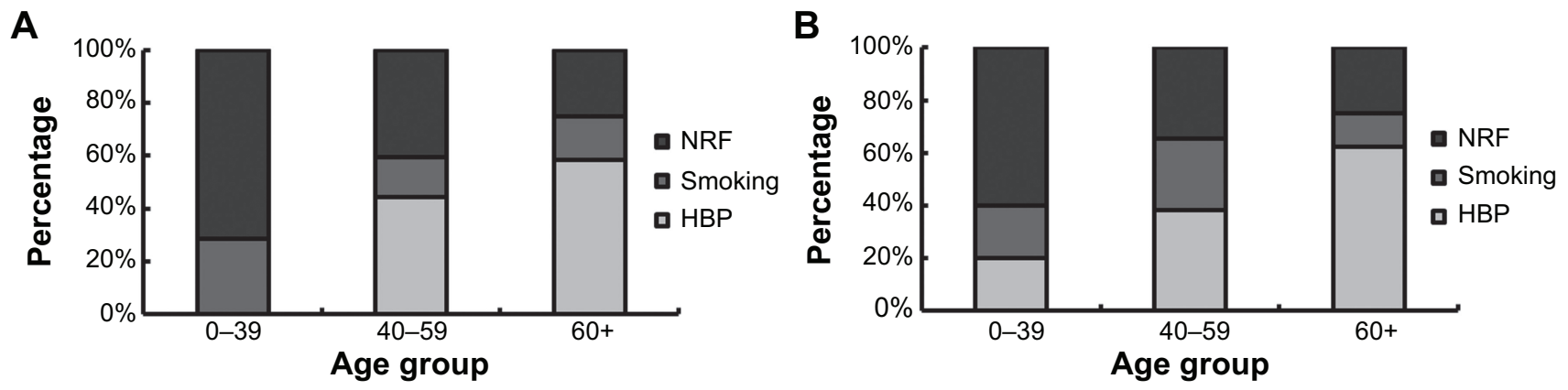

Figure I Relative prevalence of risk factors by age. (A) In patients with MirAn. (B) In nMirAn patients.

Note: Smoking refers to cigarette smoking.

Abbreviations: HBP, high blood pressure; MirAn, mirror-like intracranial aneurysms; nMirAn, non-mirror-like multiple aneurysms; NRF, no known extrinsic risk factors. 
Table 2 Hunt and Hess grade and aneurysm sites in patients with $\mathrm{SAH}$

\begin{tabular}{ll}
\hline Clinical feature & N (\%) \\
\hline Patients with SAH & $40(100)$ \\
Hunt and Hess grade, $n$ (\%) & $19(44.2)$ \\
I & $12(27.9)$ \\
III & $6(14)$ \\
IV & $3(7)$ \\
Ruptured aneurysms, $n(\%)$ & \\
One & $35(81.4)$ \\
Two & $5^{*}(11.16)$ \\
Aneurysm site, n (\%) & \\
Undetermined & $1(2.5)$ \\
Left hemisphere & $20(50)$ \\
Right hemisphere & $18(45)$ \\
Both hemispheres & I $(2.5)$ \\
Artery, n (\%) & \\
Posterior communicating arteries & $26(65.0)$ \\
Artery bifurcations & $14(35.0)$ \\
\hline
\end{tabular}

Notes: *In four of these patients, the rupture occurred again because they refused to undergo operation or interventional therapy. The fifth patient underwent open surgery after the first rupture of the left posterior communicating aneurysm, but refused to undergo surgery for the contralateral aneurysm; therefore, the right-side aneurysm ruptured and the patient underwent another surgery.

Abbreviation: SAH, subarachnoid hemorrhage. arterial bifurcation, and one (2.3\%) had aneurysms of the ophthalmic arteries. One patient (2.3\%) had multiple MirAn, with bilateral aneurysms of the middle cerebral arteries combined with bilateral aneurysms of the A3 segments of the anterior cerebral arteries.

A total of 88 aneurysms were found in the 43 MirAn patients. Of these patients, 39 (90.7\%) underwent aneurysmal clipping after craniotomy. Representative DSA images taken before and after clipping are shown in Figures 2 and 3, respectively. All surgeries were performed using a typical peritoneal approach. The remaining four patients (9.3\%) underwent interventional embolization. After the initial treatment, 19 patients (44.2\%) received additional intervention for contralateral aneurysms via a two-stage operation in which aneurysmal clipping was performed. All 39 patients who underwent aneurysmal clipping (90.7\%) were followed up and assessed for further treatment. A total of 62 aneurysms ( $70.5 \%$ of all the MirAn cases) were treated. Table 3 summarizes the treatment and follow-up details of the MirAn patients.
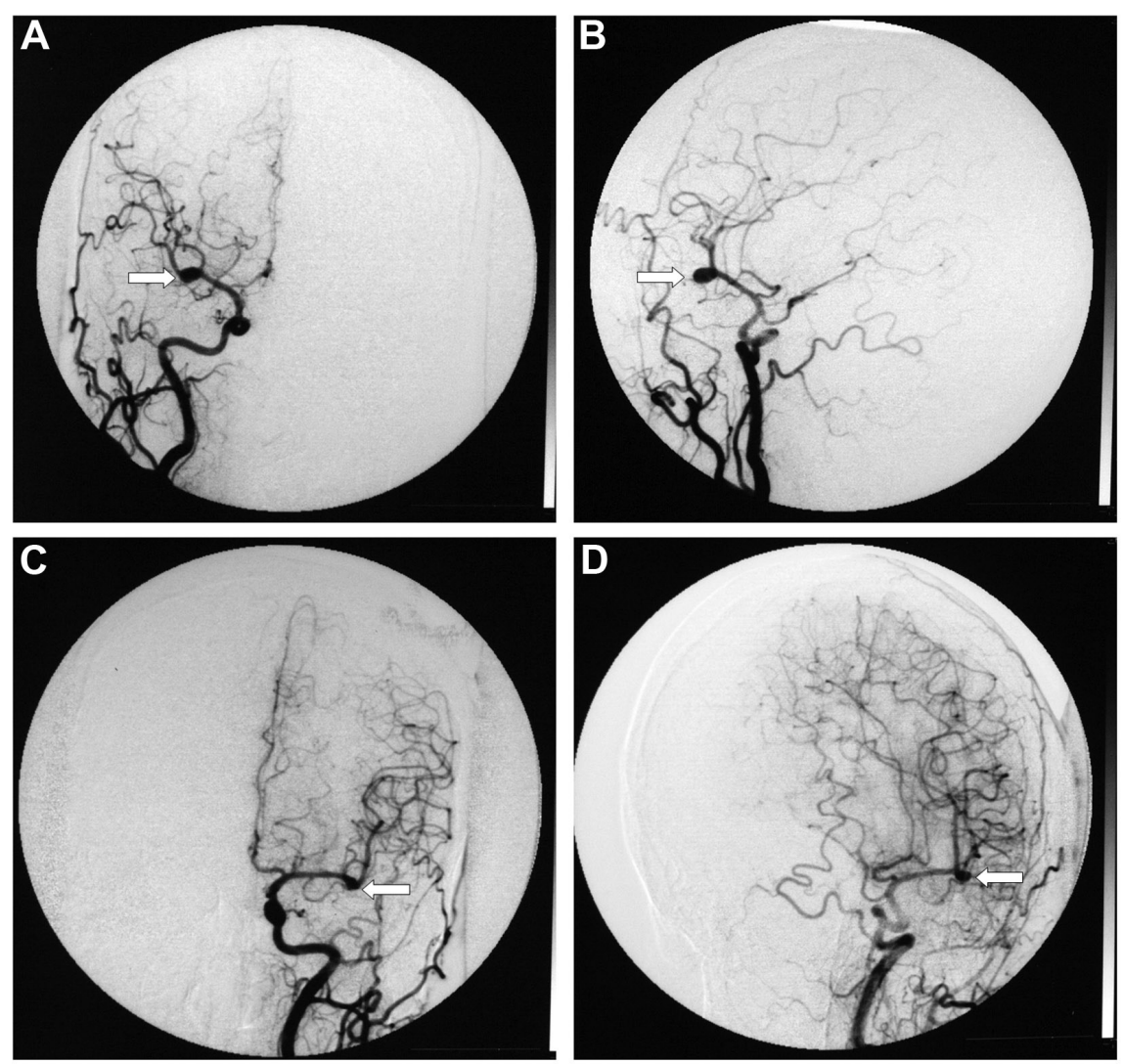

Figure 2 DSA images of both the left and right internal carotid arteries before clipping. DSA suggests that the aneurysms arise from the branches of the bilateral middle cerebral artery (indicated by arrow).

Notes: (A) Right internal carotid artery (normal position); (B) right internal carotid artery (lateral position); (C) left internal carotid artery (normal position); (D) left internal carotid artery (lateral position).

Abbreviation: DSA, digital subtraction angiography. 

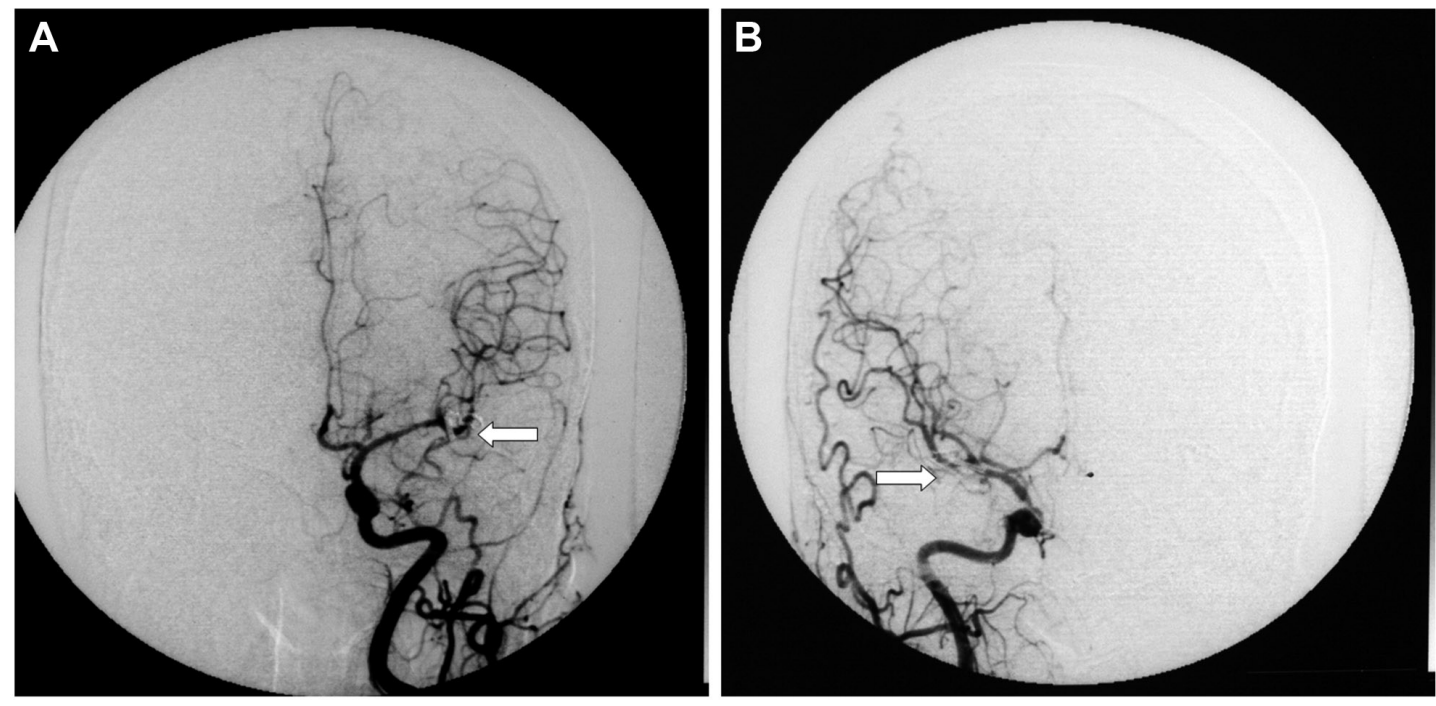

Figure 3 DSA images of the left and right internal carotid arteries after clipping. Both aneurysms from the bilateral middle cerebral artery branches are completely occluded (normal position, indicated by the arrow).

Notes: (A) Left aneurysms; (B) right aneurysms.

Abbreviation: DSA, digital subtraction angiography.

\section{Prognosis}

Of the 43 MirAn patients, four (9.3\%) were lost during follow-up. All the remaining 39 patients were followed up for 3 years. The patients were assessed according to the GOS. The results showed a good prognosis for $95.3 \%$ of the patients (GOS, 4-5 points) and bad prognosis in two patients (4.7\%), with a Hunt and Hess score of 4 for these two patients. Out of all the patients who did or did not undergo secondary treatment, none had ruptured aneurysms during follow-up.

\section{Discussion}

The aim of the present study was to explore the risk factors associated with MirAn, as well as treatment outcomes. Our findings showed that significantly more women suffered from MirAn, and that age, smoking, diabetes, and hypertension were not associated with MirAn. Further, the internal carotidposterior communicating arteries were mostly involved in MirAn. Moreover, in MirAn patients, the rupture risk was significantly higher for large aneurysms.

Table 3 Treatment and follow-up for the patients with MirAn

\begin{tabular}{ll}
\hline Treatment or follow-up & $\begin{array}{l}\text { Patients with } \\
\text { MirAn, } \mathbf{n}(\%) \mathbf{N}=\mathbf{4 3}\end{array}$ \\
\hline Total number of patients treated & $43(100)$ \\
Aneurysmal clipping & $39(90.7)$ \\
Embolization & $4(9.3)$ \\
Additional intervention via aneurysmal clipping & $19(44.2)$ \\
Follow-up and further assessment & $39(90.7)$ \\
\hline
\end{tabular}

Abbreviation: MirAn, mirror-like intracranial aneurysms.
Very few studies have compared the clinical features and treatment of MirAn patients, and most of them are case reports. ${ }^{1,4,7,8}$ To the best of our knowledge, only two other large-scale comparative studies on MirAn have been reported. One of them was by Meissner et $\mathrm{al}^{3}$ who examined the medical reports of 3,120 patients with aneurysms, out of which 376 patients (12.1\%) had MirAn. There was no significant difference in age, smoking, hypertension, and cardiac disease between MirAn and nMirAn patients, which concurs with our results. Further, although they did not examine the treatments, there was no difference in the prognosis between the MirAn and nMirAn patients. ${ }^{3}$ In the present study, prognosis of the MirAn and nMirAn groups were not compared, but MirAn patients of the present study mostly had a good prognosis (95.3\% of them), and none recurred during follow-up. Another cohort study was performed by Casimiro et $\mathrm{al}^{2}$ on 33 MirAn patients and 49 nMirAn patients. The study investigated age, smoking, and hypertension between the two groups and concluded that the lack of risk factors in young MirAn patients might indicate genetic predisposition to the disease. In addition, in the present study, the male to female ratio of the MirAn patients decreased with age, which was previously reported by Casimiro et al. ${ }^{2}$ However, in the present study, only one patient in the SingAn group had a family history of aneurysms. Therefore, this suggests that the genetic predisposition to MirAn might be multigenic or be the results of complex gene-environment interactions. A recent review reports that a number of genes are likely to be associated 
with intracranial aneurysms. ${ }^{10}$ Further studies in patients with MirAn are needed to assess the presence of mutations or polymorphisms in these patients.

In the present study, the patients with diagnosed MirAn represented $38.1 \%$ of all patients with multiple intracranial aneurysms. Our data were consistent with a previous study by Casimiro et al ${ }^{2}$ in which $40.2 \%$ (33/82) of all intracranial multiple aneurysms were diagnosed as MirAn. Further, previous studies have shown that women are more vulnerable to intracranial aneurysms compared with men. ${ }^{1,2}$ Our study also confirmed that among patients with $\mathrm{SAH}$, the male to female ratio was 40:60 (1.0:1.5).,11 The male to female ratio in our study was 1.0:1.3 in the SingAn group and 1.0:5.1 in the MirAn group, which confirms the findings of previous studies. ${ }^{5,11}$ The most significant difference between sexes was observed in the 40-59-year-old patients, while differences in the $>60$ age group were not significant. The highest incidence of MirAn was observed in the 50-59 age group. The incidence of MirAn according to age was similar to that of nMirAn. However, when compared with the SingAn group, the age with peak incidence was relatively higher in the MirAn group. Similar to our findings, previous studies have suggested that the incidence of multiple intracranial aneurysms was higher among women in their $50 \mathrm{~s}$, while the incidence of SingAn was higher in women aged $\leq 20$ years. ${ }^{12,13}$ In the present study, we observed that the incidence of MirAn was higher in women aged $>29$ years, with the highest incidence noted in the 40-59-year age group. This difference was less apparent after the age of 60 years; this may be attributable to the hormonal changes that occur after menopause. This observation was in agreement with another study that showed a correlation between menopause and higher incidence of cerebrovascular diseases. ${ }^{14}$ While the peak incidence of SingAn in women was in the 40-49-year age group, MirAn occurred more frequently in the 50-59-year age group, as previously observed. ${ }^{2}$ This is probably due to a strong genetic predisposition that promotes vascular fragility in rupture, even in the absence of other risk factors, as previously suggested. ${ }^{2}$

It has been suggested that smoking and hypertension are major risk factors for MirAn. ${ }^{12}$ However, we did not observe any association of MirAn with smoking or hypertension in our study. Casimiro et $\mathrm{al}^{2}$ also reported that $80 \%$ of MirAn patients and $20 \%$ of nMirAn patients under the age of 40 did not have extrinsic risk factors. Another study examining intracranial aneurysms in siblings found that the risk of rupture was greater when patients with unruptured aneurysms had siblings with aneurysmal SAH and/or anterior communicating artery aneurysms. ${ }^{15}$ Thus, a congenital predisposition, rather than degenerative causes, might be more likely to contribute to the pathogenesis of MirAn., ${ }^{2,15}$ In addition, in our study, none of the patients with MirAn had a family history of aneurysms, suggesting that there might be no dominant predisposing inherited factor involved in MirAn. However, since the sample size of MirAn patients was relatively small, this needs to be confirmed in larger studies.

MirAn commonly occur in intracranial anterior circulation, and mainly affect the internal carotid-posterior communicating arteries and the middle cerebral arteries. ${ }^{16}$ In our study, 40 patients (93\%) had aneurysms at these sites. However, MirAn typically do not occur in anterior communicating arteries. ${ }^{17}$

The presence of two pairs of affected arteries is extremely rare in MirAn patients. We encountered only one such case in our study, and only one other similar case has ever been reported in the literature. ${ }^{7}$ Both patients were women. The patient of the present study had bilateral aneurysms of the middle cerebral arteries combined with bilateral aneurysms of the A3 segments of the anterior cerebral arteries, which were different from the previously reported case. ${ }^{7}$ However, the rarity of these cases makes difficult any comparison.

Ruptured intracranial aneurysms are usually associated with a high mortality rate: $50 \%$ of the patients die shortly after hemorrhage, and $25 \%$ of the patients suffer from permanent neurological dysfunction; in addition, the remaining $25 \%$ have an increased risk of recurrent bleeding. ${ }^{18}$ Therefore, treatment should be initiated as soon as possible in hemorrhaging patients. ${ }^{19,20}$ In order to treat bleeding MirAn, the initial treatment should focus primarily on the hemisphere with the bleeding aneurysm. ${ }^{17,21,22}$ Our findings suggested that the rupture risk was significantly higher for large aneurysms, which is consistent with the findings of previous studies. ${ }^{11,23}$ Therefore, it may be a good strategy to perform primary treatment on the hemisphere harboring the larger aneurysm. Previous studies have suggested that the risk factors for aneurysm rupture are the same on either side. ${ }^{24,25}$ Thus, in the case of rupture on one side, the risk of rupture on the other side is high. Only one patient in our study had ruptured aneurysms on both sides. This patient underwent open surgery after the first rupture of the left posterior communicating aneurysm, but refused to undergo surgery for the contralateral aneurysm; therefore, the right-side aneurysm ruptured, and the patient had to undergo another surgery.

In the present study, we did not compare the treatments and prognosis for MirAn and nMirAn patients. However, the majority of the patients underwent aneurysmal clipping 
as the first recommended treatment option. Interventional embolization was performed in four patients because they had a Hunt and Hess grade of IV or above or myocardial infarction, severe pulmonary infection, renal insufficiency, or coagulation abnormalities, or because they were reluctant to undergo craniotomy.

One limitation of this study was the lack of long-term follow-up, including DSA and CT angiography at regular intervals. Had these examinations been conducted, changes in the untreated aneurysms or the presence of new aneurysms might have been detected. In addition, the retrospective nature of the present study could have led to a selection bias. Patients and family were asked for medical history on admission. However, no specific imaging and laboratory testing were performed for the detection of specific diseases or syndromes affecting the risk of aneurysms, unless specific symptoms were present and needed treatments.

In conclusion, a higher incidence of MirAn was observed in women. Additionally, the internal carotid-posterior communicating arteries and middle cerebral arteries were the most common affected sites in MirAn. The present study provides new insights in the epidemiology and pathology of this rare disorder.

\section{Disclosure}

The authors have no conflict of interests to report.

\section{References}

1. Baccin CE, Krings T, Alvarez H, Ozanne A, Lasjaunias P. Multiple mirror-like intracranial aneurysms. Report of a case and review of the literature. Acta Neurochir (Wien). 2006;148(10):1091-1095; discussion 1095.

2. Casimiro MV, McEvoy AW, Watkins LD, Kitchen ND. A comparison of risk factors in the etiology of mirror and nonmirror multiple intracranial aneurysms. Surg Neurol. 2004;61(6):541-545.

3. Meissner I, Torner J, Huston J 3rd, et al. Mirror aneurysms: a reflection on natural history. J Neurosurg. 2012;116(6):1238-1241.

4. Dinc C, Iplikcioglu AC, Bikmaz K, Kosdere S, Navruz Y. Distal anterior cerebral artery mirror aneurysms and middle cerebral artery aneurysms. Neurol Med Chir (Tokyo). 2006;46(9):438-440.

5. Phillips LH 2nd, Whisnant JP, O'Fallon WM, Sundt TM Jr. The unchanging pattern of subarachnoid hemorrhage in a community. Neurology. 1980;30(10):1034-1040.

6. Salunke P, Malik V, Yogesh N, Khandelwal NK, Mathuriya SN. Mirrorlike aneurysms of proximal anterior cerebral artery: report of a case and review of literature. Br J Neurosurg. 2010;24(6):686-687.
7. Sousa J, Iyer V, Roberts G. 'Mirror image' distal anterior cerebral artery aneurysms. A case report of two patients with review of literature. Acta Neurochir (Wien). 2002;144(9):933-935; discussion 935.

8. Yamada K, Nakahara T, Kishida K, et al. Multiple "mirror" aneurysms involving intracavernous carotid arteries and vertebral arteries: case report. Surg Neurol. 2000;54(5):361-365.

9. Hunt WE, Hess RM. Surgical risk as related to time of intervention in the repair of intracranial aneurysms. J Neurosurg. 1968;28(1):14-20.

10. Tromp G, Weinsheimer S, Ronkainen A, Kuivaniemi H. Molecular basis and genetic predisposition to intracranial aneurysm. Ann Med. 2014:1-10.

11. Juvela S, Poussa K, Porras M. Factors affecting formation and growth of intracranial aneurysms: a long-term follow-up study. Stroke. 2001;32(2):485-491.

12. Kongable GL, Lanzino G, Germanson TP, et al. Gender-related differences in aneurysmal subarachnoid hemorrhage. J Neurosurg. 1996;84(1):43-48.

13. Lanzino G, Kassell NF, Germanson TP, et al. Age and outcome after aneurysmal subarachnoid hemorrhage: why do older patients fare worse? J Neurosurg. 1996;85(3):410-418.

14. Wenger NK, Speroff L, Packard B. Cardiovascular health and disease in women. N Engl J Med. 1993;329(4):247-256.

15. Kasuya H, Onda H, Takeshita M, Hori T, Takakura K. Clinical features of intracranial aneurysms in siblings. Neurosurgery. 2000;46(6): 1301-1305; discussion 1305-1306.

16. Kaminogo M, Yonekura M, Shibata S. Incidence and outcome of multiple intracranial aneurysms in a defined population. Stroke. 2003;34(1):16-21.

17. Cervoni L, Delfini R, Santoro A, Cantore G. Multiple intracranial aneurysms: surgical treatment and outcome. Acta Neurochir (Wien). 1993;124(2-4):66-70.

18. Peters DG, Kassam AB, Feingold E, et al. Molecular anatomy of an intracranial aneurysm: coordinated expression of genes involved in wound healing and tissue remodeling. Stroke. 2001;32(4):1036-1042.

19. Broderick JP, Brott TG, Duldner JE, Tomsick T, Leach A. Initial and recurrent bleeding are the major causes of death following subarachnoid hemorrhage. Stroke. 1994;25(7):1342-1347.

20. Fogelholm R, Hernesniemi J, Vapalahti M. Impact of early surgery on outcome after aneurysmal subarachnoid hemorrhage. A populationbased study. Stroke. 1993;24(11):1649-1654.

21. Li G, Yin L, Sun J. [Microsurgical treatment of multiple intracranial aneurysms]. Zhonghua wai ke za zhi. 2001;39(9):667-668. Chinese.

22. Rinne J, Hernesniemi J, Niskanen M, Vapalahti M. Management outcome for multiple intracranial aneurysms. Neurosurgery. 1995;36(1):31-37; discussion 37-38.

23. Juvela S, Porras M, Heiskanen O. Natural history of unruptured intracranial aneurysms: a long-term follow-up study. J Neurosurg. 1993;79(2):174-182.

24. Ellamushi HE, Grieve JP, Jager HR, Kitchen ND. Risk factors for the formation of multiple intracranial aneurysms. J Neurosurg. 2001;94(5):728-732.

25. Qureshi AI, Suarez JI, Parekh PD, et al. Risk factors for multiple intracranial aneurysms. Neurosurgery. 1998;43(1):22-26; discussion 26-27.

\section{Dovepress}

\section{Publish your work in this journal}

Neuropsychiatric Disease and Treatment is an international, peerreviewed journal of clinical therapeutics and pharmacology focusing on concise rapid reporting of clinical or pre-clinical studies on a range of neuropsychiatric and neurological disorders. This journal is indexed on PubMed Central, the 'PsycINFO' database and CAS, and is the official journal of The International Neuropsychiatric Association (INA). The manuscript management system is completely online and includes a very quick and fair peer-review system, which is all easy to use. Visit http://www.dovepress.com/testimonials.php to read real quotes from published authors. 